\title{
Exceptional Minimal Surfaces Whose Gauss Images Have Constant Curvature
}

\author{
Makoto SAKAKI \\ Hirosaki University \\ (Communicated by T. Nagano) \\ Dedicated to Professor Y. Hatakeyama on his 60th birthday
}

\section{Introduction.}

Let $M$ be a minimal surface in the $N$-dimensional Euclidean space $\boldsymbol{R}^{\boldsymbol{N}}$ with Gaussian curvature $K(\leqq 0)$ with respect to the induced metric $d s^{2}$. We consider the Gauss map from $M$ to the Grassmann manifold $G_{2, N}$ of 2-planes in $R^{N}$, where $G_{2, N}$ may be identified with the complex quadric $Q_{N-2}$ in the $(N-1)$-dimensional complex projective space $C P^{N-1}$ of constant holomorphic sectional curvature 2. Then the metric $d \hat{s}_{0}^{2}$ on $M$ induced by the Gauss map is $-K d s^{2}$, which is degenerate at points where $K=0$ (see [7]). Let $\hat{K}_{0}$ denote the Gaussian curvature of $M$ with respect to $d \hat{s}_{0}^{2}$, which is the Gaussian curvature of the Gauss image of $M$. Lawson [7], Hoffman and Osserman [4] discussed minimal surfaces in $\boldsymbol{R}^{N}$ with constant $\hat{K}_{0}$. In particular, they showed that if $M$ is a minimal surface lying fully in $\boldsymbol{R}^{N}$ with constant $\hat{K}_{0}$, then $\hat{K}_{0}$ must be of the form $2 / m$ for some positive integer $m$, and $m+1 \leqq N \leqq 2 m+2$. Some examples of minimal surfaces in $\boldsymbol{R}^{N}$ with constant $\hat{K}_{0}$ are given in [3].

In [5] Johnson studied a class of minimal surfaces in space forms, which are called exceptional minimal surfaces. First, in this paper, we discuss exceptional minimal surfaces in $\boldsymbol{R}^{N}$ with constant $\hat{K}_{0}$.

THEOREM 1. Let $\boldsymbol{M}$ be an exceptional minimal surface lying fully in $\boldsymbol{R}^{\boldsymbol{N}}$ with constant $\hat{K}_{0}$. Then $\hat{K}_{0}=1 / n$ when $N=2 n+1$, and $\hat{K}_{0}=2 / n$ when $N=2 n+2$.

REMARK 1. (i) We will also show that for every positive integer $n$, there are exceptional minimal surfaces lying fully in $R^{2 n+1}$ with $\hat{K}_{0}=1 / n$, and in $R^{2 n+2}$ with $\hat{K}_{0}=2 / n$.

(ii) By Theorem 1 and [3], we can find that there are non-exceptional minimal surfaces in $\boldsymbol{R}^{N}$ with constant $\hat{K}_{0}$.

Next, we deal with the case where the ambient spaces are other space forms. Let $M$ be a minimal surface in the $N$-dimensional simply connected space form $X^{N}(c)$ of constant curvature $c$. We denote by $K(\leqq c)$ the Gaussian curvature of $M$ with respect 
to the induced metric $d s^{2}$. We consider Obata's Gauss map from $M$ to the space of all totally geodesic 2-subspaces in $X^{N}(c)$ (see [8]). The metric $d \hat{s}_{c}^{2}$ on $M$ induced by the Gauss map is $(c-K) d s^{2}$, which is degenerate at points where $K=c$ (see [8]). Let $\hat{K}_{c}$ denote the Gaussian curvature of $M$ with respect to $d \hat{s}_{c}^{2}$, which is the Gaussian curvature of the Gauss image of $M$. We discuss exceptional minimal surfaces in $X^{6}(c)$ with constant $\hat{K}_{c}$, where $c \neq 0$.

THEOREM 2. Let $M$ be an exceptional minimal surface in $X^{6}(c)$ with constant $\hat{K}_{c}$, where $c>0$. Then $M$ has constant curvature $c / 3, c / 6$ or 0 .

THEOREM 3. There are no exceptional minimal surfaces in $X^{6}(c)$ with constant $\hat{K}_{c}$, where $c<0$.

REMARK 2. Bryant [1] classified minimal surfaces with constant curvature in space forms. Minimal surfaces with positive constant curvature in $X^{N}(c)$, where $c>0$, are parts of minimal 2-spheres. So they are exceptional (see [5] and [2]). In [9] we noted that for every positive integer $n$, there are flat exceptional minimal surfaces lying fully in $X^{2 n+1}(c)$, where $c>0$.

\section{Exceptional minimal surfaces.}

In this section, we follow [5] and recall the definition of exceptional minimal surfaces. Suppose $M$ is a minimal surface in $X^{N}(c)$. Assume that $M$ lies fully in $X^{N}(c)$, namely, does not lie in a totally geodesic submanifold of $X^{N}(c)$. Let the integer $n$ be given by $N=2 n+1$ or $2 n+2$, and let indices have the following ranges:

$$
1 \leqq i, j \leqq 2, \quad 3 \leqq \alpha \leqq N, \quad 1 \leqq A, B \leqq N .
$$

Let $\tilde{e}_{A}$ be a local orthonormal frame field on $X^{N}(c)$, and let $\tilde{\theta}_{A}$ be the coframe dual to $\tilde{e}_{A}$. Then $d \tilde{\theta}_{A}=\sum_{B} \tilde{\omega}_{A B} \wedge \tilde{\theta}_{B}$, where $\tilde{\omega}_{A B}$ are the connection forms on $X^{N}(c)$.

Suppose that $e_{i}$ is a local orthonormal frame field on $M$ and that the frame $\tilde{e}_{A}$ is chosen so that on $M, e_{i}=\tilde{e}_{i}$ and $\tilde{e}_{\alpha}$ are normal to $M$. When forms and vectors on $X^{N}(c)$ are restricted to $M$, let them be denoted by the same symbol without tilde: $\theta_{A}=$ $\left.\tilde{\theta}_{A}\right|_{M}, \omega_{A B}=\left.\tilde{\omega}_{A B}\right|_{M}$ and $e_{A}=\left.\tilde{e}_{A}\right|_{M}$. Then $\omega_{\alpha i}=\sum_{j} h_{\alpha i j} \theta_{j}$, where $h_{\alpha i j}$ are the coefficients of the second fundamental form of $M$.

Let $T_{x} M$ and $T_{x} X^{N}(c)$ denote the tangent space of $M$ and $X^{N}(c)$, respectively, at a point $x$. Curves on $M$ through $x$ have their first derivatives at $x$ in $T_{x} M$, but higher order derivatives will have components normal to $M$. The space spanned by the derivatives of order up to $r$ is called the $r$-th osculating space of $M$ at $x$, denoted $T_{x}^{(r)} M$.

The $r$-th normal space of $M$ at $x$, denoted $\operatorname{Nor}_{x}^{(r)} M$, is the orthogonal complement of $T_{x}^{(r)} M$ in $T_{x}^{(r+1)} M$. At generic points of $M$, the dimension of $\operatorname{Nor}_{x}^{(r)} M$ is 2 when $1 \leqq r \leqq n-1$, and the dimension of $\operatorname{Nor}_{x}^{(n)} M$ is 1 or 2 , depending on whether $N$ is odd or even. Those normal spaces that have dimension 2 are called the normal planes of $M$. 
Let $\beta_{N}$ denote the number of normal planes possessed by $M$ at generic points: $\beta_{N}=n-1$ if $N=2 n+1$, and $\beta_{N}=n$ if $N=2 n+2$.

Choose the normal vectors $e_{\alpha}$ so that $\operatorname{Nor}_{x}^{(r)} M$ is spanned by $\left\{e_{2 r+1}, e_{2 r+2}\right\}$, where $1 \leqq r \leqq \beta_{N}$. When $N=2 n+1$, $\operatorname{Nor}_{x}^{(n)} M$ is spanned by $\left\{e_{2 n+1}\right\}$. Set $\varphi=\theta_{1}+\sqrt{-1} \theta_{2}$. Then there are $H_{\alpha}$ such that $H_{\alpha}=h_{\alpha 11}+\sqrt{-1} h_{\alpha 12}$ for $\alpha=3$ and 4 , for each $r$ such that $2 \leqq r \leqq \beta_{N}$

$$
H_{2 r-1} \omega_{\alpha, 2 r-1}+H_{2 r} \omega_{\alpha, 2 r}=H_{\alpha} \bar{\varphi}
$$

where $\alpha=2 r+1$ and $2 r+2$, and when $N=2 n+1$

$$
H_{2 n-1} \omega_{2 n+1,2 n-1}+H_{2 n} \omega_{2 n+1,2 n}=H_{2 n+1} \bar{\varphi}
$$

(see [5]).

The $r$-th normal plane, $\operatorname{Nor}_{x}^{(r)} M$, of $M$ is called exceptional if $H_{2 r+2}= \pm \sqrt{-1} H_{2 r+1}$. The minimal surface $M$ is called exceptional if all of its normal planes are exceptional. Note that when $N=2 n+1, \operatorname{Nor}_{x}^{(n)} M$ is a line, not a plane, and the notion of exceptionality does not apply. So, every minimal surface in $X^{3}(c)$ is exceptional.

\section{A lemma.}

Let $\left(M, d s^{2}\right)$ be a 2-dimensional Riemannian manifold with Gaussian curvature $K<c$. We denote by $\Delta$ the Laplacian of $\left(M, d s^{2}\right)$. Set

$$
\begin{gathered}
A_{0}^{c}=1 / 2, \quad A_{1}^{c}=c-K, \\
A_{p+1}^{c}= \begin{cases}A_{p}^{c}\left[\Delta \log \left(A_{p}^{c}\right)+A_{p}^{c} / A_{p-1}^{c}-2(p+1) K\right], & \text { if } A_{p}^{c}>0, \\
0, & \text { otherwise } .\end{cases}
\end{gathered}
$$

Let $\hat{K}_{c}$ be the Gaussian curvature of $M$ with respect to the metric $d \hat{s}_{c}^{2}=(c-K) d s^{2}$. Then

$$
\hat{K}_{c}=\frac{K}{c-K}-\frac{1}{2(c-K)} \Delta \log (c-K) .
$$

Now suppose that $c=0$ and $\hat{K}_{0}=2 / m$, where $m$ is a positive integer. Then by (2), we have

$$
\Delta \log (-K)=2\left(1+\frac{2}{m}\right) K
$$

LEMMA. Under the hypothesis above,

$$
A_{p}^{0}=p((p-1) !)^{2}\left\{\prod_{k=1}^{p-1}\left(\frac{2}{k}-\frac{2}{m}\right)\right\}(-K)^{p} \quad \text { for } 2 \leqq p \leqq m+1,
$$

and 


$$
A_{p}^{0}=0 \quad \text { for } p \geqq m+1 .
$$

Proof. By (1) and (3), we have

$$
\begin{aligned}
A_{2}^{0} & =(-K)[\Delta \log (-K)-2 K-4 K] \\
& =2\left(2-\frac{2}{m}\right)(-K)^{2},
\end{aligned}
$$

and

$$
\begin{aligned}
A_{3}^{0} & =2\left(2-\frac{2}{m}\right)(-K)^{2}\left[2 \Delta \log (-K)-2\left(2-\frac{2}{m}\right) K-6 K\right] \\
& =12\left(2-\frac{2}{m}\right)\left(1-\frac{2}{m}\right)(-K)^{3}
\end{aligned}
$$

So the lemma is true for $p=2$ and 3 . Assume that the lemma is true for $p$ and $p+1$, where $2 \leqq p \leqq m-1$. Then by (1), (3) and the assumption,

$$
\begin{aligned}
A_{p+2}^{0}= & (p+1)(p !)^{2}\left\{\prod_{k=1}^{p}\left(\frac{2}{k}-\frac{2}{m}\right)\right\}(-K)^{p+1} \\
& \times\left[(p+1) \Delta \log (-K)-p(p+1)\left(\frac{2}{p}-\frac{2}{m}\right) K-2(p+2) K\right] \\
= & (p+2)((p+1) !)^{2}\left\{\prod_{k=1}^{p+1}\left(\frac{2}{k}-\frac{2}{m}\right)\right\}(-K)^{p+2} .
\end{aligned}
$$

So the lemma is true for $p+2$. Therefore, by induction, the lemma is true for $2 \leqq p \leqq m+1$. Thus we have $A_{m+1}^{0}=0$, and by (1) we have $A_{p}^{0}=0$ for $p \geqq m+1$.

Q.E.D.

\section{Proof of Theorem 1.}

Proof of Theorem 1. Let $d s^{2}$ and $K$ be as in Section 0 . We assume that $K<0$ in the theorem because $\hat{K}_{0}$ cannot be defined at points where $K=0$. Let $\Delta$ and $A_{p}^{0}$ be as in Section 1. By [4], $\hat{K}_{0}=2 / m$ for some positive integer $m$. So the equation (3) and Lemma are valid.

When $N=2 n+1$, by Theorem A of [5], $A_{p}^{0} \geqq 0$ for $1 \leqq p \leqq n$ with equality only at isolated points, and the metric $\left(A_{n}^{0}\right)^{1 /(n+1)} d s^{2}$ is flat at points where $A_{p}^{0}>0$ for $1 \leqq p \leqq n$. So by Lemma, we find that $m \geqq n$ and

$$
A_{n}^{0}=n((n-1) !)^{2}\left\{\prod_{k=1}^{n-1}\left(\frac{2}{k}-\frac{2}{m}\right)\right\}(-K)^{n} \text {. }
$$

Using the lemma in Section 3 of [5] and the equation (3), we have 


$$
0=\Delta \log \left(A_{n}^{0}\right)-2(n+1) K=\left(\frac{4 n}{m}-2\right) K .
$$

Thus we have $m=2 n$, and $\hat{K}_{0}=1 / n$.

When $N=2 n+2$, by Theorem A of [5], $A_{p}^{0} \geqq 0$ for $1 \leqq p \leqq n$ with equality only at isolated points, and $A_{n+1}^{0}=0$ identically. So by Lemma, we have $m=n$, and $\hat{K}_{0}=2 / n$.

Q.E.D.

We shall show the fact in Remark 1 (i). Let $\left(M, d s^{2}\right)$ be a 2-dimensional Riemannian manifold with Gaussian curvature $K<0$. Let $\Delta, A_{p}^{0}$ and $\hat{K}_{0}$ be defined as in Section 1 .

First suppose that $\hat{K}_{0}=1 / n$, where $n$ is a positive integer. We note that there are such 2-dimensional Riemannian manifolds. Then the equation (3) and Lemma are valid for $m=2 n$. So $A_{p}^{0}>0$ for $p \leqq 2 n$. Using Lemma and (3) with $m=2 n$, we have

$$
\Delta \log \left(A_{n}^{0}\right)-2(n+1) K=0 .
$$

By the lemma in Section 3 of [5], the metric $\left(A_{n}^{0}\right)^{1 /(n+1)} d s^{2}$ is flat. By Theorem B of [5], $\left(M, d s^{2}\right)$ can be realized locally as an exceptional minimal surface lying fully in $\boldsymbol{R}^{2 n+1}$. Therefore, for every positive integer $n$, there are exceptional minimal surfaces lying fully in $R^{2 n+1}$ with $\hat{K}_{0}=1 / n$.

Next suppose that $\hat{K}_{0}=2 / n$, where $n$ is a positive integer. Then the lemma is valid for $m=n$. So $A_{p}^{0}>0$ for $p \leqq n$ and $A_{n+1}^{0}=0$. By Theorem B of [5], $\left(M, d s^{2}\right)$ can be realized locally as an exeptional minimal surface lying fully in $\boldsymbol{R}^{2 n+2}$. Therefore, for every positive integer $n$, there are exceptional minimal surfaces lying fully in $\boldsymbol{R}^{2 n+2}$ with $\hat{K}_{0}=2 / n$.

\section{Proof of Theorems 2 and 3.}

In this section we prove the following proposition. Combining the proposition with [1], we have Theorems 2 and 3.

Proposition. Let $M$ be an exceptional minimal surface in $X^{6}(c)$ with constant $\hat{K}_{c}$, where $c \neq 0$. Then $M$ has constant curvature.

Proof. Let $d s^{2}$ and $K$ be as in Section 0. We assume that $K<c$ in the proposition because $\hat{K}_{c}$ cannot be defined at points where $K=c$. Let $\Delta$ and $A_{p}^{c}$ be as in Section 1 . We assume that $\hat{K}_{c}=a$. Then by (2), we have

$$
\Delta \log (c-K)=2\{(a+1) K-c a\} .
$$

By (1) and (4),

$$
\begin{aligned}
A_{2}^{c} & =(c-K)[\Delta \log (c-K)+2(c-K)-4 K] \\
& =2(c-K)\{(a-2) K-c(a-1)\} .
\end{aligned}
$$


Set $M_{1}=\left\{x \in M ; A_{2}^{c}>0\right\}$. By (1), (4) and (5),

(6) $A_{3}^{c}=A_{2}^{c}[\Delta \log (c-K)+\Delta \log \{(a-2) K-c(a-1)\}+2\{(a-2) K-c(a-1)\}-6 K]$

$$
=A_{2}^{c}[\Delta \log \{(a-2) K-c(a-1)\}+2\{2(a-2) K-c(2 a-1)\}]
$$

on $M_{1}$.

Now suppose that $M$ lies fully in $X^{N}(c)$ where $3 \leqq N \leqq 6$. When $N=3$, by Theorem $A$ and the lemma in Section 3 of [5],

$$
\Delta \log (c-K)=4 K .
$$

By (4) and (7), we can see that $K$ is constant. When $N=4, A_{2}^{c}=0$ identically by Theorem A of [5]. Then by (5), we can see that $K$ is constant.

When $N=5$, by Theorem A of [5], $M_{1}$ is $M$ minus isolated points and the metric $\left(A_{2}^{c}\right)^{1 / 3} d s^{2}$ is flat on $M_{1}$. Using the lemma in Section 3 of [5], the equations (4) and (5), we have

$$
\begin{aligned}
0 & =\Delta \log \left(A_{2}^{c}\right)-6 K \\
& =\Delta \log (c-K)+\Delta \log \{(a-2) K-c(a-1)\}-6 K \\
& =\Delta \log \{(a-2) K-c(a-1)\}+2\{(a-2) K-c a\}
\end{aligned}
$$

on $M_{1}$. By (8) we can see that $a \neq 2$. By (4) and (8), we have

$$
\Delta K=F(K)=b_{0}+b_{1} K+b_{2} K^{2}+b_{3} K^{3}
$$

and

$$
|\nabla K|^{2}=G(K)=b_{4}+b_{5} K+b_{6} K^{2}+b_{7} K^{3}+b_{3} K^{4}
$$

on $M_{1}$ and, by continuity, on $M$, where

$$
\begin{array}{cc}
b_{0}=-\frac{2 c^{2} a\left(2 a^{2}-6 a+5\right)}{a-2}, & b_{1}=2 c\left(6 a^{2}-9 a-1\right), \\
b_{2}=-12 a(a-2), & b_{3}=\frac{2(a-2)(2 a-1)}{c}, \\
b_{4}=\frac{2 c^{3} a(a-1)(2 a-3)}{a-2}, & b_{5}=-\frac{2 c^{2} a\left(8 a^{2}-24 a+17\right)}{a-2}, \\
b_{6}=2 c\left(12 a^{2}-18 a+1\right), & b_{7}=-2\left(8 a^{2}-16 a+3\right) .
\end{array}
$$

If $K$ is not constant, then

$$
G K+\left(F-G^{\prime}\right)\left(F-\frac{1}{2} G^{\prime}\right)+G\left(F^{\prime}-\frac{1}{2} G^{\prime \prime}\right)=0,
$$


where the prime denotes the differentiation with respect to $K$ (see for example [6, p. 136]). The left-hand side of (9) is a polynomial of $K$ such that the coefficient of $K^{5}$ is $-16(a-2)(2 a-1) / c$ and the constant term is $-8 c^{4} a(a-1)(5 a-3) /(a-2)$. So it is a nontrivial polynomial. Thus $K$ must be constant, which is a contradiction. Therefore, $K$ is constant.

When $N=6$, by Theorem A of [5], $M_{1}$ is $M$ minus isolated points and $A_{3}^{c}=0$ identically. By (6) we have

$$
\Delta \log \{(a-2) K-c(a-1)\}+2\{2(a-2) K-c(2 a-1)\}=0
$$

on $M_{1}$. By (10) we can see that $a \neq 2$. By (4) and (10), we have

$$
\Delta K=P(K)=d_{0}+d_{1} K+d_{2} K^{2}+d_{3} K^{3}
$$

and

$$
|\nabla K|^{2}=Q(K)=d_{4}+d_{5} K+d_{6} K^{2}+d_{7} K^{3}+d_{3} K^{4}
$$

on $M_{1}$ and, by continuity, on $M$, where

$$
\begin{gathered}
d_{0}=-\frac{2 c^{2}\left(3 a^{3}-9 a^{2}+8 a-1\right)}{a-2}, \quad d_{1}=2 c\left(9 a^{2}-15 a+2\right), \\
d_{2}=-6(a-2)(3 a-1), \quad d_{3}=\frac{6(a-1)(a-2)}{c}, \\
d_{4}=\frac{2 c^{3}(a-1)\left(3 a^{2}-5 a+1\right)}{a-2}, \quad d_{5}=-\frac{2 c^{2}\left(12 a^{3}-39 a^{2}+35 a-7\right)}{a-2}, \\
d_{6}=2 c\left(18 a^{2}-33 a+10\right), \quad d_{7}=-2\left(12 a^{2}-29 a+13\right) .
\end{gathered}
$$

If $K$ is not constant, then

$$
Q K+\left(P-Q^{\prime}\right)\left(P-\frac{1}{2} Q^{\prime}\right)+Q\left(P^{\prime}-\frac{1}{2} Q^{\prime \prime}\right)=0
$$

(similar to (9)). The left-hand side of (11) is a polynomial of $K$ such that the coefficient of $K^{5}$ is $6(a-1)(a-2)(3 a-14) / c$ and the constant term is $-2 c^{4}(a-1)\left(9 a^{3}+12 a^{2}-\right.$ $14 a+1) /(a-2)$. When $a=1$, the coefficient of $K$ is $16 c^{3} \neq 0$. So the left-hand side of (11) is a nontrivial polynomial. Thus $K$ must be constant, which is a contradiction. Therefore, $K$ is constant.

Q.E.D.

Minimal 2-spheres in $X^{N}(c)$, where $c>0$, are always exceptional (see [5] and [2]). So, by Theorem 2 , we have the following:

COROllaRY. Let $M$ be a minimal 2-sphere in $X^{6}(c)$ with constant $\hat{K}_{c}$, where $c>0$. Then $M$ has constant curvature $c / 3$ or $c / 6$. 


\section{References}

[ 1 ] R. BRyant, Minimal surfaces of constant curvature in $S^{n}$, Trans. Amer. Math. Soc., 290 (1985), $259-271$.

[2] S. S. CHERN, On the minimal immersions of the two-sphere in a space of constant curvature, Problems in Analysis, Princeton Univ. Press, 1970, pp. 27-40.

[ 3 ] M. FuJIKı, On the Gauss map of minimal surfaces immersed in $\boldsymbol{R}^{n}$, Kodai Math. J., 9(1986), $44-49$.

[4] D. Hoffman and R. Osserman, The Geometry of the Generalized Gauss Map, Mem. Amer. Math. Soc., 236 (1980).

[5] G. D. Johnson, An intrinsic characterization of a class of minimal surfaces in constant curvature manifolds, Pacific J. Math., 149 (1991), 113-125.

[6] K. Kenmotsu, Minimal surfaces with constant curvature in 4-dimensional space forms, Proc. Amer. Math. Soc., 89 (1983), 133-138.

[7] H. B. LAwson, Some intrinsic characterizations of minimal surfaces, J. Analyse Math., 24 (1971), 151-161.

[8] M. Obata, The Gauss map of immersions of Riemannian manifolds in spaces of constant curvature, J. Diff. Geom., 2 (1968), 217-223.

[9] M. SAKAKI, Exceptional minimal surfaces with the Ricci condition, Tsukuba J. Math., 16 (1992), 161-167.

Present Address:

Department of Mathematics, Faculty of Science, Hirosaki University HiRosaki 036, JAPAN 